\title{
Composição química do sedimento e de folhas das espécies do manguezal do estuário do Rio São Mateus, Espírito Santo, Brasil
}

\author{
ELAINE BERNINI ${ }^{1,4}$, MARIA AMÉLIA B. DA SILVA ${ }^{1}$, TANIA MARA S. DO CARMO ${ }^{2}$ e \\ GERALDO ROGÉRIO F. CUZZUOL ${ }^{3}$
}

(recebido: 30 de setembro de 2004; aceito: 23 de novembro de 2006)

\begin{abstract}
Chemical composition of sediments and leaves of mangrove species at the São Mateus river estuary, Espírito Santo State, Brazil). We analyzed nutrient concentrations in leaves and sediments, and element accumulation capacity in foliar tissues of Avicennia germinans (L.) Stearn., Avicennia schaueriana Staft \& Leechm., Laguncularia racemosa (L.) Gaertn. f. and Rhizophora mangle L. in four sites along the São Mateus River estuary mangrove, Espírito Santo State, SE-Brazil. In sediment, elements decrease following the sequence: $\mathrm{Mg}>\mathrm{Ca}>\mathrm{Fe}>\mathrm{K}>\mathrm{Mn}>\mathrm{P}>\mathrm{Zn}>\mathrm{Cu}$ and showed inter and intraespecific variation. Avicennia leaves showed the greatest $\mathrm{N}, \mathrm{K}$ and $\mathrm{Mg}$ concentrations and lowest Ca concentrations. Rhizophora mangle presented the greatest Mn concentration, and Laguncularia racemosa the greatest Fe level. Sediment nutrient concentrations reflected the influence of granulometry in this compartment. Nutrient accumulation in leaves varied according to species and study site but it was not correlated with sediment concentrations. Results confirmed the mangrove role as a biogeochemistry barrier blocking heavy metal up to tropical coastal areas. However, it is necessary periodic sampling so that it can be possible a better understanding of the nutrient dynamic.
\end{abstract}

Key words - heavy metals, leaves, mangrove, nutrients

RESUMO - (Composição química do sedimento e de folhas das espécies do manguezal do estuário do Rio São Mateus, Espírito Santo, Brasil). As concentrações de nutrientes das folhas e do sedimento e a capacidade de acumulação de elementos químicos dos tecidos foliares das espécies Avicennia germinans (L.) Stearn., Avicennia schaueriana Staft \& Leechm., Laguncularia racemosa (L.) Gaertn. f. e Rhizophora mangle L. foram analisadas em quatro estações de estudo no manguezal do estuário do Rio São Mateus, no Estado do Espírito Santo. De modo geral, os elementos determinados no sedimento seguiram a ordem: $\mathrm{Mg}>\mathrm{Ca}>\mathrm{Fe}>\mathrm{K}>\mathrm{Mn}>\mathrm{P}>\mathrm{Zn}>\mathrm{Cu}$, apresentando variações inter e intraespecíficas. No tecido foliar, as espécies de Avicennia apresentaram maiores teores de N, K e Mg e menores concentrações de Ca. Rhizophora mangle exibiu maior concentração de Mn e Laguncularia racemosa, maior teor de Fe em relação às demais espécies. Os resultados demonstraram que a concentração de nutrientes do sedimento refletiu a influência da granulometria neste compartimento. $\mathrm{O}$ acúmulo de nutrientes nas folhas variou de acordo com a espécie e estação de estudo, mas não refletiu as concentrações do sedimento. Os dados confirmaram o papel do manguezal como barreira biogeoquímica ao trânsito de metais pesados. Entretanto, torna-se desejável uma amostragem periódica para um melhor entendimento da dinâmica de nutrientes.

Palavras-chave - folhas, manguezal, metais pesados, nutrientes

\section{Introdução}

O conhecimento da dinâmica e da estrutura dos ecossistemas demanda a compreensão dos teores de nutrientes, sua distribuição nas plantas e no solo, processos de ciclagem e fluxos de entrada e de saída (Hay \& Lacerda 1984). A comunidade vegetal

\footnotetext{
1. Universidade Federal do Espírito Santo (UFES), Pólo Universitário de São Mateus, Departamento de Biologia. Avenida João XXIII, 1544, Boa Vista, 29931-220 São Mateus, ES, Brasil.

2. Universidade Federal do Espírito Santo, Centro de Ciências Humanas e Naturais (CCHN), Laboratório de Bioecologia do Manguezal.

3. Universidade Federal do Espírito Santo, CCHN, Departamento de Biologia.

4. Autor para correspondência: bernini@uenf.br
}

representa papel chave na circulação dos elementos, sendo, portanto, essencial determinar a sua composição química.

Todas as plantas requerem a mesma rede básica de nutrientes inorgânicos, sendo vários elementos químicos utilizados por diferentes plantas para fins essencialmente similares (Fitter \& Hay 1987). Entretanto, as espécies diferem amplamente em sua concentração absoluta e nas proporções relativas dos diferentes elementos, uma vez que a eficiência de absorção de nutrientes e a preferência por um determinado elemento dependem de um conjunto de fatores internos e externos (Waisel 1972, Larcher 2000). No ecossistema manguezal, a absorção de determinados cátions parece depender da salinidade. A absorção de $\mathrm{Ca}$, por exemplo, é controlada pelo nível de $\mathrm{Na}$ no sedimento (Waisel 1972, Joshi et al. 1975), o que, 
provavelmente, também ocorre em relação à absorção de Mg. A absorção de N e K, por outro lado, parece ser controlada fisiologicamente (Lacerda 1986).

No sedimento do manguezal, os teores de nutrientes geralmente variam ao longo da zona intertidal. As variações são atribuídas principalmente à freqüência de inundação pelas marés (Lacerda et al. 1985) e ao grau de saturação do sedimento, que influencia o potencial redox e pode afetar a forma e a disponibilidade de elementos químicos como Cu, Fe e Zn (Ball 1988). No entanto, as concentrações dos elementos nas folhas de mangue não se correlacionam com o conteúdo de nutrientes do sedimento, revelando a absorção diferencial dos íons pelas plantas (Lacerda et al. 1986, Medina et al. 2001). Dessa forma, o manguezal pode atuar como eficiente barreira biogeoquímica ao trânsito de metais pesados em áreas costeiras, através da imobilização de metais nos sedimentos sob formas não biodisponíveis que, juntamente com certas adaptações fisiológicas típicas das árvores de mangue, reduzem sensivelmente a absorção de metais pesados pelas plantas (Aragon et al. 1986, Silva et al. 1990, Lacerda 1997).

No Brasil, dados referentes à composição química de folhas e sedimentos de mangue foram publicados principalmente para os estados da Bahia, Pará, Rio de Janeiro e São Paulo (Lamberti 1969, Lacerda et al. 1985, Cuzzuol \& Campos 2001, Medina et al. 2001, Bernini 2003). No Estado do Espírito Santo, Almeida (2001) determinou o conteúdo de nutrientes na fração folhas da serapilheira do manguezal do Canal da Passagem, em Vitória.

Os objetivos deste estudo foram: 1) determinar as concentrações de nutrientes do sedimento e das folhas de espécies do manguezal do Estuário do Rio São Mateus, com o propósito de fornecer maiores informações sobre a composição química das plantas de mangue no Estado do Espírito Santo; e 2) determinar a capacidade de acumulação de elementos químicos dos tecidos foliares de espécies presentes neste manguezal.

\section{Material e métodos}

Área de estudo - O estudo foi realizado no manguezal do Estuário do Rio São Mateus, localizado à Sudeste do Município de Conceição da Barra (figura 1A), Estado do Espírito Santo, Brasil ( $18^{\circ} 35^{\prime}$ S e $39^{\circ} 44^{\prime}$ W). O clima da região é tropical úmido. Considerando a década de 90 , a temperatura média foi de $23,9^{\circ} \mathrm{C}$ e a precipitação média anual de $1.372 \mathrm{~mm}$ (fonte dos dados: Instituto Capixaba de Pesquisa, Assistência Técnica e Extensão Rural, Incaper, Linhares, ES, 19²4' S e $40^{\circ} 04^{\prime}$ W). O estuário está sob o regime micromareal, com marés semidiurnas. Baseado em dados do Terminal de Barra do Riacho, ES (19²4' S e 4003' W), no período entre 1998 e 2000, a média a das marés foi de $0,8 \mathrm{~m}$ (Diretoria de Hidrografia e Navegação, Ministério da Marinha).

O manguezal do Estuário do Rio São Mateus ocupa área aproximada de $11 \mathrm{~km}^{2}$ (Vale 1999), cuja floresta é constituída pelas espécies Avicennia germinans (L.) Stearn., Avicennia schaueriana Staft \& Leechm., Laguncularia racemosa (L.) Gaertn. f. e Rhizophora mangle L., além das espécies associadas Acrostichum aureum L. e Hibiscus pernambucensis Arruda (Silva et al. 2005). Este manguezal tem sido alvo de freqüentes ações de degradação como assoreamento, deposição de lixo, lançamento de efluentes domésticos, atividade extrativista de árvores para obtenção de lenha e aterros para ocupações irregulares.

O estudo foi conduzido nas quatro estações de estudo selecionadas por Silva et al. (2005), que caracterizaram a estrutura da vegetação do manguezal do estuário do Rio São Mateus. Os parâmetros estruturais analisados encontram-se resumidos na tabela 1 . As estações 1 e 2 situaram-se no estuário inferior e as estações 3 e 4 no estuário médio (figura 1B). Considerando os tipos fisiográficos de florestas de mangue classificados por Lugo \& Snedaker (1974) e Cintrón et al. (1985) e posteriormente modificados por SchaefferNovelli et al. (2000), os bosques analisados são do tipo franja, pois ocorrem na borda de um ambiente em contato com o corpo d'água.

Em cada estação de estudo foram selecionadas cinco árvores de cada espécie presente a $10 \mathrm{~m}$ da margem do rio. $\mathrm{Na}$ estação 1 foram analisadas as espécies Laguncularia racemosa e Rhizophora mangle; na estação 2, Avicennia schaueriana, Laguncularia racemosa e Rhizophora mangle; na estação 3, Laguncularia racemosa e Rhizophora mangle; e na estação 4, Avicennia germinans. Para cada árvore foi coletada uma amostra de sedimento $(0-15 \mathrm{~cm})$ entre as raízes e cerca de 40 folhas adultas e verdes, provenientes do $3^{\circ}$ ou 4 nó, dos galhos expostos ao sol. As coletas foram realizadas em maio de 1999. As amostras foram acondicionadas em sacos plásticos previamente etiquetados e enviadas ao Laboratório de Análise Química e Física do Incaper para posterior análises.

Em cada estação de estudo foram realizadas, in situ, três medidas de $\mathrm{pH}$ (potenciômetro modelo pHTestr $2^{\mathrm{TM}}$ ) e salinidade (refratômetro modelo 10049 American Optical, com precisão de \pm 1 ) da água intersticial ( $30 \mathrm{~cm}$ de profundidade) entre as raízes de um indivíduo de cada espécie presente.

A granulometria do sedimento foi determinada pelo método do densímetro. As classificações texturais seguiram a metodologia de Shepard, segundo Suguio (1973), baseadas nos percentuais de areia, silte e argila. Para efeito de cálculos, as porções areia grossa e areia fina foram somadas, sendo consideradas como fração areia. A matéria orgânica (fração $<2 \mathrm{~mm}$ ) foi determinada pelo método indireto (digestão úmida com dicromato de potássio e ácido sulfúrico).

Os elementos químicos do sedimento foram determinados para a fração $<2 \mathrm{~mm}$, de acordo com metodologia 
Tabela 1. Resumo dos parâmetros estruturais determinados nas quatro estações de estudo analisadas (E1, E2, E3 e E4) no manguezal do Estuário do Rio São Mateus (Silva et al. 2005). (DAP = diâmetro à altura do peito).

Table 1. Summary of the structural parameters determined in the four study stations analyzed (E1, E2, E3 and E4) in the mangrove at São Mateus River estuary (Silva et al. 2005). ( $\mathrm{DAP}=$ diameter to breast height).

\begin{tabular}{lccccc}
\hline Estação & $\begin{array}{c}\text { Altura } \\
(\mathrm{m})^{*}\end{array}$ & $\begin{array}{c}\text { DAP } \\
\text { médio }(\mathrm{cm})\end{array}$ & $\begin{array}{c}\text { Área basal viva } \\
\left(\mathrm{m}^{2} \mathrm{ha}^{-1}\right)\end{array}$ & $\begin{array}{c}\text { Densidade } \\
\left(\text { ind ha }^{-1}\right)\end{array}$ & Espécie dominante \\
\hline E1 & $5,6 \pm 1,5$ & 8,1 & 7,5 & 1.450 & Rhizophora mangle L. \\
E2 & $5,4 \pm 1,8$ & 8,2 & 7,2 & 1.225 & $\begin{array}{c}\text { Rhizophora mangle L. } \\
\text { E3 }\end{array}$ \\
E4 & $12,9 \pm 1,8$ & 14,0 & 17,9 & 1.100 & Laguncularia racemosa (L.) Gaertn. f. \\
Avicennia germinans $($ L. $)$ Stearn.
\end{tabular}

* = média \pm desvio padrão.

$*=$ mean \pm standard deviation.

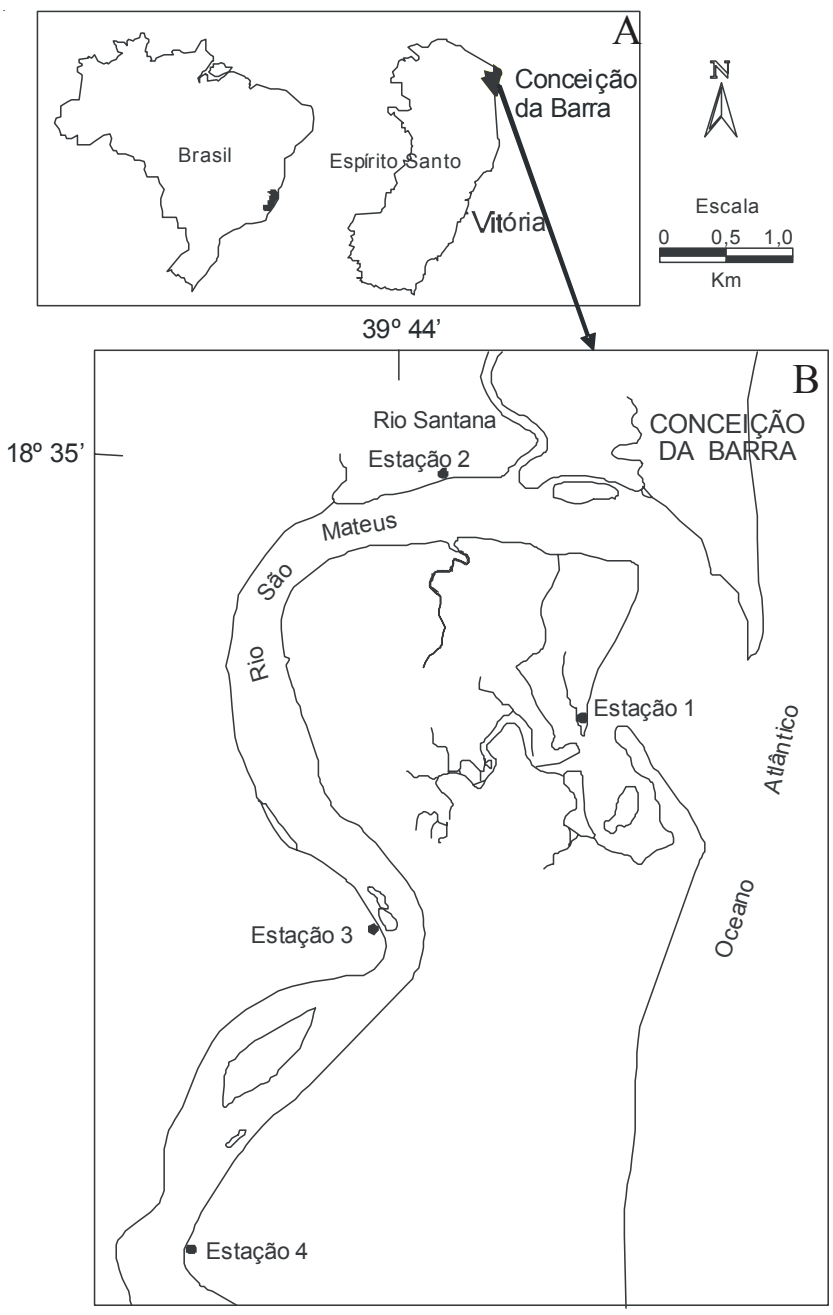

Figura 1. A. Conceição da Barra, Estado do Espírito Santo, Brasil (18 35' S e 39 44' W). B. Localização das estações de estudo analisadas no manguezal do Estuário do Rio São Mateus.

Figure 1. A. Conceição da Barra, Espírito Santo State, Brazil $\left(18^{\circ} 35^{\prime} \mathrm{S}\right.$ e $\left.39^{\circ} 44^{\prime} \mathrm{W}\right)$. B. Location of the study stations in the mangrove at São Mateus River estuary. descrita em Embrapa (1997). A extração das frações móveis de $\mathrm{P}, \mathrm{K}, \mathrm{Fe}, \mathrm{Mn}, \mathrm{Zn}$ e Cu foi realizada com solução de $\mathrm{HCl}$ 0,05 $\mathrm{N}+\mathrm{H}_{2} \mathrm{SO}_{4} 0,025 \mathrm{~N}$, na relação solo:extrator 1:5. Adeterminação do $\mathrm{K}$ foi feita por Fotometria de Chama, do $\mathrm{P}$ por Colorimetria $(725 \mathrm{~nm})$ e dos demais elementos, por espectrofotometria de absorção atômica convencional (EAA). $\mathrm{O} \mathrm{Ca}$ e o $\mathrm{Mg}$ trocáveis foram extraídos com $\mathrm{KCl} 1 \mathrm{~N}$ e determinados pelo método complexométrico com o emprego de EDTA (titulometria).

Os teores de nutrientes do tecido foliar foram determinados segundo metodologia descrita em Sarruge \& Haag (1974) e Silva (1981). O nitrogênio total foi determinado pela digestão Microkjeldahl. Para a extração de $\mathrm{Ca}, \mathrm{Cu}, \mathrm{Fe}, \mathrm{K}$, $\mathrm{Mg}, \mathrm{Mn}, \mathrm{P}, \mathrm{S}$ e Zn utilizou-se digestão nítrico-perclórica. A determinação do fósforo total foi feita por Colorimetria $(725 \mathrm{~nm})$ e os demais elementos por EAA. Após digestão, o enxofre foi quantificado por determinação gravimétrica, que se baseia na precipitação do enxofre pelo cloreto de bário, na forma de sulfato de bário.

A concentração foliar foi dividida pela concentração do sedimento para determinar o fator de concentração (FC), segundo Salisbury \& Ross (1992). Os dados foram submetidos a análise de variância (one-way ANOVA) sendo a diferença mínima significativa determinada pelo Teste de Tukey em nível de $5 \%$ de probabilidade. O coeficiente de correlação de Pearson foi calculado para os nutrientes analisados nas folhas e no sedimento, sendo aplicado o Teste de Student para testar a significância das correlações. A análise estatística foi realizada através do programa Statistic.

\section{Resultados}

Água intersticial e sedimento - A salinidade da água intersticial variou entre $9 \%$ e $34 \%$, sendo maior nas estações 1 e 2 (tabela 2). Não houve diferença interespecífica significativa dentro de cada estação, mas constatou-se diferença intraespecífica entre as estações de estudo, visto que a salinidade foi inferior na estação 
3 para Laguncularia racemosa (L.) Gaertn. f. e Rhizophora mangle L. O pH variou de levemente ácido a neutro, sem diferença interespecífica dentro de cada estação (tabela 2). Na estação 2, a água intersticial de Rhizophora mangle apresentou valores mais elevados de $\mathrm{pH}$, em relação às demais estações.

Os percentuais médios das frações inorgânicas e a classificação textural do sedimento são apresentados na tabela 2. Na estação 1 o sedimento mostrou características mais grosseiras que as das demais estações, sendo classificado como arenoso. Na estação 2, os sedimentos de Laguncularia racemosa e Rhizophora mangle foram classificados como areia síltico-lamosa. Nesta estação, a quantidade de argila foi significativamente maior no sedimento de Avicennia schaueriana Staft \& Leechm., caracterizando um sedimento do tipo lama arenosa. Nas estações 3 e 4, o sedimento foi classificado como lama síltico-arenosa, pois houve predominância de sedimentos finos (silte e argila). Considerando todas as estações de estudo, o percentual médio de matéria orgânica do sedimento foi considerado reduzido, variando entre $0,48 \%$ na estação 1 e 2,34\% na estação 4 (tabela 2). Esta variável não diferiu significativamente entre as espécies dentro de cada estação.

Com relação aos nutrientes determinados no sedimento, de um modo geral, os elementos seguiram a ordem: $\mathrm{Mg}>\mathrm{Ca}>\mathrm{Fe}>\mathrm{K}>\mathrm{Mn}>\mathrm{P}>\mathrm{Zn}>\mathrm{Cu}$ (tabela 3). O sedimento da estação 1 apresentou teores mais reduzidos de nutrientes em relação às demais estações (tabela 3). Analisando-se os sedimentos por espécie (tabela 4) observa-se que Rhizophora mangle exibiu maior concentração de $\mathrm{Cu}$ em relação à Laguncularia racemosa na estação 1. Na estação 2, os teores de K, P e Zn foram inferiores no sedimento de Rhizophora mangle, enquanto que a concentração de Mn foi superior no sedimento de Avicennia schaueriana. $\mathrm{Na}$ estação 3, Laguncularia racemosa exibiu maior concentração de $\mathrm{Ca}$ em relação à Rhizophora mangle. Foram verificadas diferenças intraespecíficas entre as estações de estudo, sendo que os teores de $\mathrm{Ca}, \mathrm{K}, \mathrm{Mg}$, $\mathrm{Fe}$ e $\mathrm{Cu}$, nos sedimentos de Laguncularia racemosa e Rhizophora mangle, foram maiores nas estações 2 e 3 . O sedimento de Rhizophora mangle também revelou maiores concentrações de $\mathrm{P}$, Mn e Zn na estação 3. O sedimento de Laguncularia racemosa mostrou maior teor de $\mathrm{Mn}$ na estação 3 (tabela 4).

Folhas - A concentração geral de macronutrientes nas folhas das espécies seguiu a ordem: $\mathrm{N}>\mathrm{K}>\mathrm{Mg}>\mathrm{S}>$ $\mathrm{Ca}>\mathrm{P}>\mathrm{Fe}>\mathrm{Mn}>\mathrm{Zn}>\mathrm{Cu}$ para Avicennia germinans
(L.) Stearn.; $\mathrm{N}>\mathrm{Mg}>\mathrm{K}>\mathrm{S}>\mathrm{Ca}>\mathrm{P}>\mathrm{Fe}>\mathrm{Mn}>\mathrm{Zn}$ $>\mathrm{Cu}$ para Avicennia schaueriana; $\mathrm{N}>\mathrm{Ca}>\mathrm{Mg}>\mathrm{K}$ $>\mathrm{S}>\mathrm{P}>\mathrm{Fe}>\mathrm{Mn}>\mathrm{Zn}>\mathrm{Cu}$ para Laguncularia racemosa; e $\mathrm{N}>\mathrm{K}>\mathrm{Ca}>\mathrm{Mg}>\mathrm{S}>\mathrm{P}>\mathrm{Mn}>\mathrm{Fe}>\mathrm{Zn}$ $>\mathrm{Cu}$ para Rhizophora mangle (figura 2).

Com relação aos macronutrientes foi possível identificar dois grupos distintos (figura 2A). O primeiro grupo, constituído por Avicennia germinans e Avicennia schaueriana, demonstrou maiores teores de $\mathrm{N}$ e $\mathrm{Mg}$ e menores concentrações de $\mathrm{Ca}$ em relação ao segundo grupo formado por Laguncularia racemosa e Rhizophora mangle. Analisando-se separadamente cada estação (tabela 5), verifica-se que na estação 1 , Rhizophora mangle exibiu maior concentração de K e Laguncularia racemosa, maior de $\mathrm{P}$. Na estação 2, Avicennia schaueriana apresentou maiores teores de $\mathrm{N}, \mathrm{Mg}$ e $\mathrm{S}$ e menor concentração de $\mathrm{Ca}$ em relação às demais espécies. Nesta mesma estação, Rhizophora mangle mostrou menor teor de $\mathrm{P}$ e Laguncularia racemosa, menor de K. Na estação 3, Rhizophora mangle destacou-se pela maior concentração de $\mathrm{Mg}$, enquanto que Laguncularia racemosa apresentou valores superiores de $\mathrm{N}$ e $\mathrm{P}$.

Quanto aos micronutrientes (tabela 6, figura 2B), nas estações 1, 2 e 3, Rhizophora mangle destacou-se por apresentar maiores concentrações de Mn e menores teores de $\mathrm{Zn}$ e $\mathrm{Cu}$ (exceto na estação 2), em relação às demais espécies. Na estação 3, Laguncularia racemosa apresentou maiores concentrações de $\mathrm{Fe}$ e $\mathrm{Zn}$.

Foram observadas diferenças intraespecíficas nas concentrações de macro e micronutrientes entre as diversas estações de estudo (tabelas 5, 6). A concentração de $\mathrm{Cu}$ nas folhas de Rhizophora mangle foi superior na estação 3. Nas folhas de Laguncularia racemosa, os teores de $\mathrm{Ca}, \mathrm{Mg}$ e $\mathrm{Zn}$ foram maiores na estação 1. Esta espécie apresentou maior concentração de $\mathrm{K}$ e menor teor de $\mathrm{S}$ na estação 3.

A capacidade de acúmulo de nutrientes variou de acordo com a espécie e estação de estudo (tabela 7). Os fatores de concentração (FC, o qual indica o grau de acumulação de elementos químicos pela planta) na estação 1 foram superiores em relação às demais estações, entretanto não se observou alguma tendência de distribuição. Os macronutrientes apresentaram acúmulo nas folhas das espécies, com destaque para $\mathrm{P}$ e K. Para os micronutrientes houve registro de baixos valores de FC, principalmente para o elemento Fe. De uma maneira geral, as espécies apresentaram acúmulo de Zn. Avicennia germinans e Avicennia schaueriana apresentaram valores semelhantes para $\mathrm{Ca}, \mathrm{Mg}, \mathrm{Fe}$ e 


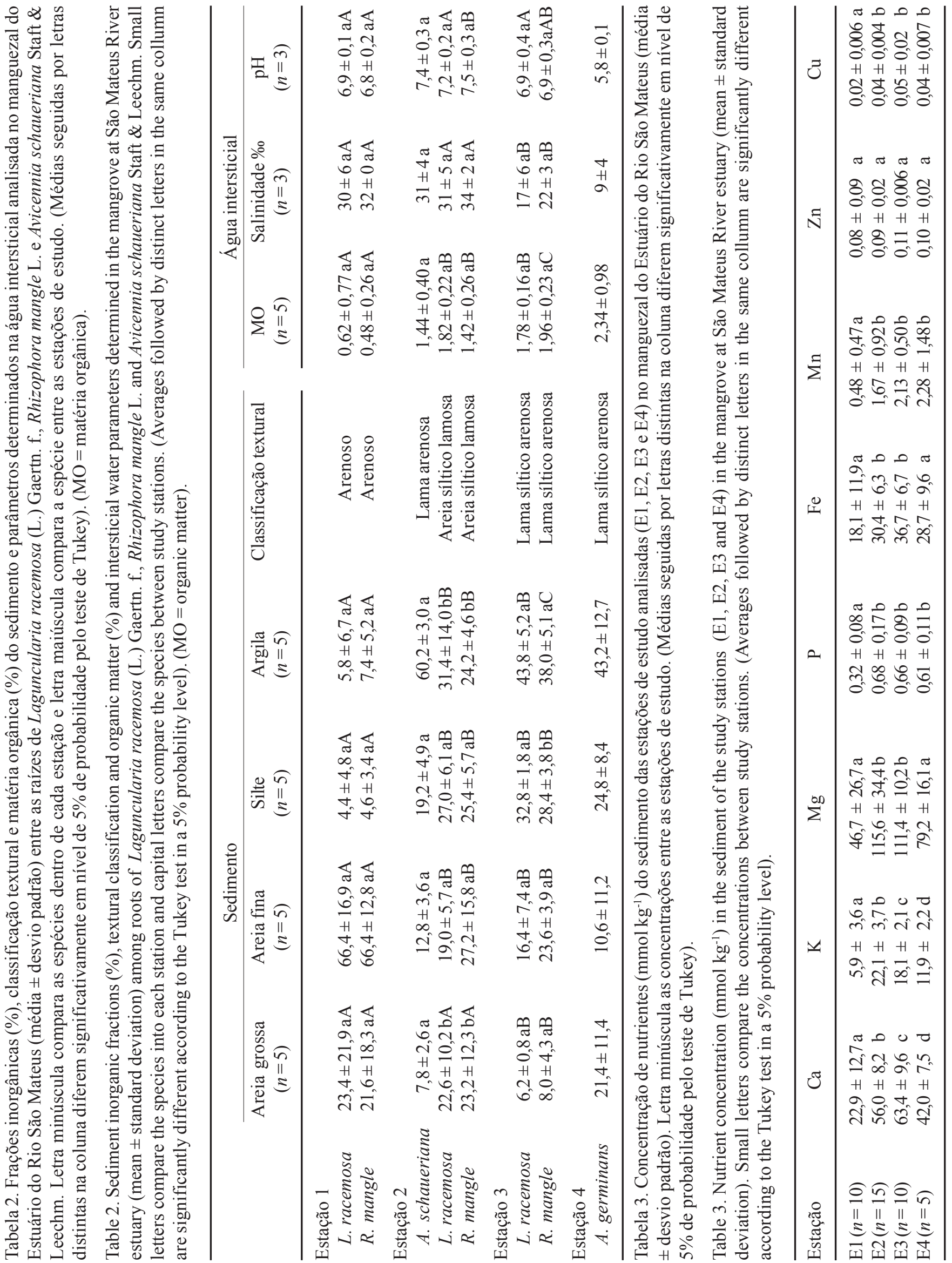




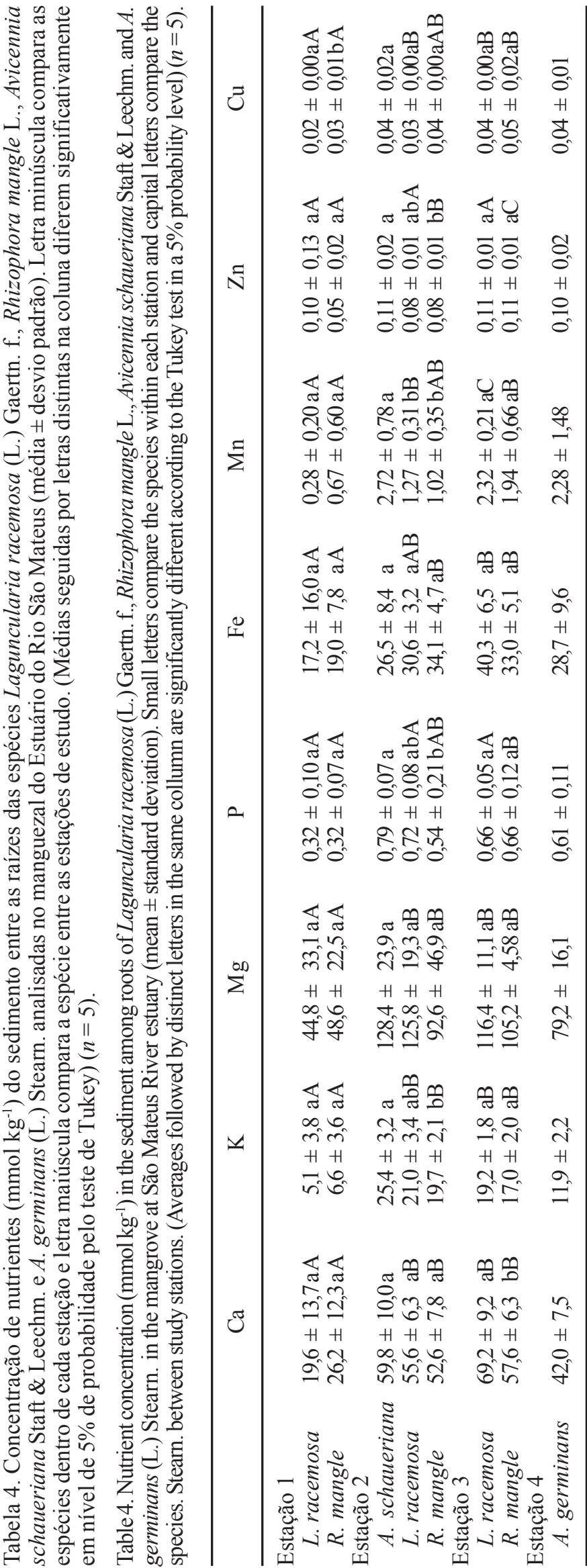

Mn. Na estação 2, os FCs de micronutrientes para Avicennia schaueriana e Laguncularia racemosa foram similares. Rhizophora mangle exibiu valores mais baixos para $\mathrm{Fe}, \mathrm{Zn}$ e $\mathrm{Cu}$ e mais elevados para $\mathrm{Mn}$ (acima de 2,0) em relação às demais espécies. As concentrações de nutrientes das folhas não se correlacionaram com as concentrações do sedimento $(P>0,05)$.

\section{Discussão}

Água intersticial e sedimento - A salinidade da água intersticial apresentou ampla variação entre as estações, sendo mais elevada nas estações 1 e 2 , pelo fato de
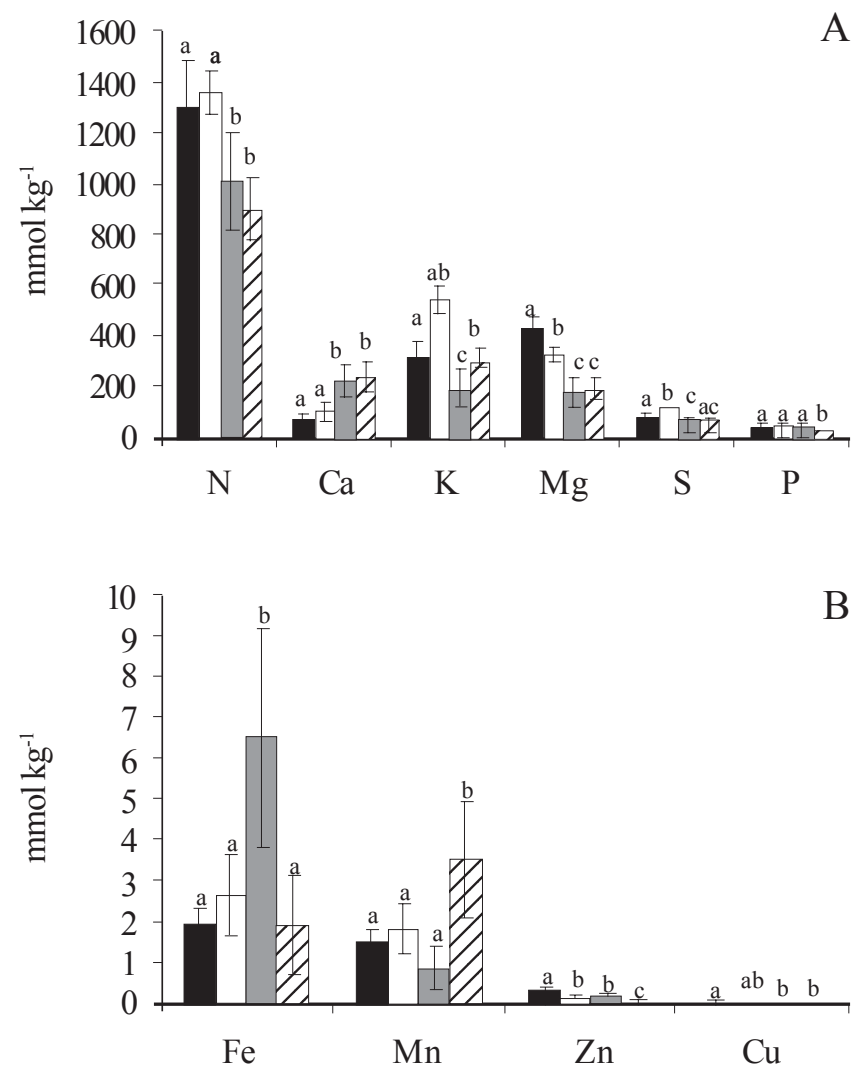

Figura 2. Valores médios de (A) macronutrientes $\left(\mathrm{mmol} \mathrm{kg}{ }^{-1}\right)$ e (B) micronutrientes $\left(\mathrm{mmol} \mathrm{kg}^{-1}\right)$ nas folhas das espécies analisadas no manguezal do Estuário do Rio São Mateus. Valores seguidos por letras distintas diferem significativamente $(P \leq 0,05)$.

Figure 2. Mean values of (A) macronutrients $\left(\mathrm{mmol} \mathrm{kg}^{-1}\right)$ and (B) micronutrients $\left(\mathrm{mmol} \mathrm{kg}^{-1}\right)$ in the species leaves from the mangrove at São Mateus River estuary. Values followed by distinct letters are significantly different $(P \leq 0,05)$.

( $\mathbf{\square}=$ Avicennia germinans (L.) Stearn.; $\square=$ Avicennia schaueriana Staft \& Leechm.; = Laguncularia racemosa (L.) Gaertn. f.; $\square=$ Rhizophora mangle L.). 
Tabela 5. Concentração de macronutrientes $\left(\mathrm{mmol} \mathrm{kg}^{-1}\right)$ nas folhas das espécies analisadas no manguezal do Estuário do Rio São Mateus (média \pm desvio padrão). Letra minúscula compara as espécies (Laguncularia racemosa (L.) Gaertn. f., Rhizophora mangle L., Avicennia schaueriana Staft \& Leechm., A. germinans (L.) Stearn.) dentro de cada estação e letra maiúscula compara a espécie entre as estações de estudo. (Médias seguidas por letras distintas na coluna diferem significativamente em nível de $5 \%$ de probabilidade pelo teste de Tukey) $(n=5)$.

Table 5. Macronutrient concentration $\left(\mathrm{mmol} \mathrm{kg}^{-1}\right)$ in the species leaves from the mangrove at São Mateus River estuary (mean \pm standard deviation). Small letters compare the species (Laguncularia racemosa (L.) Gaertn. f., Rhizophora mangle L., Avicennia schaueriana Staft \& Leechm., A. germinans (L.) Stearn.) within each station and capital letters compare the species between study stations. (Averages followed by distinct letters in the same collumn are significantly different according to the Tukey test in a $5 \%$ probability level) $(n=5)$.

\begin{tabular}{|c|c|c|c|c|c|c|}
\hline & $\mathrm{N}$ & $\mathrm{Ca}$ & $\mathrm{K}$ & $\mathrm{Mg}$ & $\mathrm{S}$ & $\mathrm{P}$ \\
\hline \multicolumn{7}{|l|}{ Estação 1} \\
\hline L. racemosa & $909,6 \pm 248,4 \mathrm{aA}$ & $272,6 \pm 73,8 \mathrm{aA}$ & $135,0 \pm 51,5 \mathrm{aA}$ & $228,7 \pm 58,9 \mathrm{aA}$ & $73,6 \pm 10,5 \mathrm{aA}$ & $39,4 \pm 5,8 \mathrm{aA}$ \\
\hline R. mangle & $849,6 \pm 100,0 \mathrm{aA}$ & $223,2 \pm 57,9 \mathrm{aA}$ & $256,3 \pm 38,6 \mathrm{bA}$ & $171,1 \pm 37,1 \mathrm{aA}$ & $71,7 \pm 11,9 \mathrm{aA}$ & $32,3 \pm 3,2 \mathrm{bA}$ \\
\hline \multicolumn{7}{|l|}{ Estação 2} \\
\hline A. schaueriana & $1359,3 \pm 65,2 \quad a$ & $97,4 \pm 14,1 \mathrm{a}$ & $326,8 \pm 61,2 \mathrm{a}$ & $538,0 \pm 86,3 \mathrm{a}$ & $112,9 \pm 10,0 \mathrm{a}$ & $48,4 \pm 4,0 \mathrm{a}$ \\
\hline L. racemosa & $979,5 \pm 83,6 \quad b A$ & $229,2 \pm 32,6 \mathrm{bAB}$ & $166,2 \pm 51,4 \mathrm{bA}$ & $178,5 \pm 31,4 \mathrm{bAB}$ & $70,5 \pm 6,1 \quad b A$ & $43,9 \pm 13,4 \mathrm{abA}$ \\
\hline R. mangle & $939,6 \pm 147,4 \mathrm{bA}$ & $258,6 \pm 55,2 \mathrm{bA}$ & $307,0 \pm 66,7 \mathrm{aA}$ & $176,0 \pm 31,7 \mathrm{bA}$ & $72,4 \pm 5,1 \quad b A$ & $30,3 \pm 1,8 \mathrm{bA}$ \\
\hline \multicolumn{7}{|l|}{ Estação 3} \\
\hline L. racemosa & $1139,5 \pm 151,6 \mathrm{aA}$ & $166,3 \pm 40,7 \mathrm{aB}$ & $259,3 \pm 53,4 \mathrm{aB}$ & $125,9 \pm 24,8 \mathrm{aB}$ & $53,6 \pm 10,4 \mathrm{aB}$ & $47,8 \pm 5,3 \mathrm{aA}$ \\
\hline R. mangle & $899,6 \pm 127,4 \mathrm{bA}$ & $229,2 \pm 70,6 \mathrm{aA}$ & $317,1 \pm 53,3$ aA & $213,9 \pm 52,7 \mathrm{bA}$ & $61,1 \pm 9,0$ aA & $32,3 \pm 3,2 \mathrm{bA}$ \\
\hline \multicolumn{7}{|l|}{ Estação 4} \\
\hline A. germinans & $1299,4 \pm 187,0$ & $67,9 \pm 14,8$ & $429,1 \pm 66,1$ & $311,8 \pm 42,1$ & 81,1 & $46,5 \pm 5,4$ \\
\hline
\end{tabular}

Tabela 6. Concentração de micronutrientes (mmol kg-1) nas folhas das espécies analisadas (Laguncularia racemosa (L.) Gaertn. f., Rhizophora mangle L., Avicennia schaueriana Staft \& Leechm., A. germinans (L.) Stearn.) no manguezal do Estuário do Rio São Mateus (média \pm desvio padrão). Letra minúscula compara as espécies dentro de cada estação e letra maiúscula compara a espécie entre as estações de estudo. (Médias seguidas por letras distintas na coluna diferem significativamente em nível de $5 \%$ de probabilidade pelo teste de Tukey) $(n=5)$

Table 6. Micronutrient concentration $\left(\mathrm{mmol} \mathrm{kg}^{-1}\right)$ in the species leaves (Laguncularia racemosa (L.) Gaertn. f., Rhizophora mangle L., Avicennia schaueriana Staft \& Leechm., A. germinans (L.) Stearn.) from the mangrove at São Mateus river estuary (mean \pm standard deviation). Small letters compare the species within each station and capital letters compare the species between study stations. (Averages followed by distinct letters in the same collumn are significantly different according to the Tukey test in a $5 \%$ probability level) $(n=5)$.

\begin{tabular}{|c|c|c|c|c|}
\hline & $\mathrm{Fe}$ & $\mathrm{Mn}$ & $\mathrm{Zn}$ & $\mathrm{Cu}$ \\
\hline \multicolumn{5}{|l|}{ Estação 1} \\
\hline L. racemosa & $5,72 \pm 3,12$ aA & $0,74 \pm 0,36$ aA & $0,25 \pm 0,07 \quad \mathrm{aA}$ & $0,04 \pm 0,01 \quad \mathrm{aA}$ \\
\hline R. mangle & $2,41 \pm 2,10 \mathrm{aA}$ & $2,60 \pm 1,27 \quad b A$ & $0,07 \pm 0,6 \quad \mathrm{bA}$ & $0,02 \pm 0,01 \quad \mathrm{bAB}$ \\
\hline \multicolumn{5}{|l|}{ Estação 2} \\
\hline A. schaueriana & $2,65 \pm 0,97 \mathrm{a}$ & $1,83 \pm 0,63 \mathrm{a}$ & $0,16 \pm 0,04 \quad \mathrm{a}$ & $0,03 \pm 0,01 \quad a$ \\
\hline L. racemosa & $5,39 \pm 1,31 \mathrm{bA}$ & $1,06 \pm 0,83$ aA & $0,15 \pm 0,03 \quad \mathrm{aB}$ & $0,03 \pm 0,01 \quad a b A$ \\
\hline R. mangle & $1,69 \pm 0,39 \mathrm{aA}$ & $3,33 \pm 0,74 \quad \mathrm{bA}$ & $0,06 \pm 0,01 \quad \mathrm{bA}$ & $0,02 \pm 0,00 \quad \mathrm{bA}$ \\
\hline \multicolumn{5}{|l|}{ Estação 3} \\
\hline L. racemosa & $8,46 \pm 2,66 \mathrm{aA}$ & $0,75 \pm 0,20 \quad \mathrm{aA}$ & $0,18 \pm 0,03 \mathrm{aAB}$ & $0,03 \pm 0,02 \quad \mathrm{aA}$ \\
\hline R. mangle & $1,59 \pm 0,21 \mathrm{bA}$ & $4,63 \pm 1,48 \quad \mathrm{bA}$ & $0,07 \pm 0,01 \quad \mathrm{bA}$ & $0,03 \pm 0,02 \quad \mathrm{aB}$ \\
\hline \multicolumn{5}{|l|}{ Estação 4} \\
\hline A. germinans & $1,98 \pm 0,34$ & $1,51 \pm 0,29$ & $0,34 \pm 0,05$ & $0,06 \pm 0,03$ \\
\hline
\end{tabular}


Tabela 7. Médias dos fatores de concentração das espécies analisadas no manguezal do Estuário do Rio São Mateus ( $n=5)$.

Table 7. Concentration factors of the species from the mangrove at São Mateus River estuary $(n=5)$.

\begin{tabular}{lcccccccc}
\hline & $\mathrm{Ca}$ & $\mathrm{K}$ & $\mathrm{Mg}$ & $\mathrm{P}$ & $\mathrm{Fe}$ & $\mathrm{Mn}$ & $\mathrm{Zn}$ & $\mathrm{Cu}$ \\
\hline $\begin{array}{l}\text { Estação 1 } \\
\quad \text { Laguncularia racemosa (L.) Gaertn. f. }\end{array}$ & 19,6 & 45,0 & 7,8 & 139,3 & 0,75 & 5,10 & 7,84 & 2,18 \\
$\quad$ Rhizophora mangle L. & 10,8 & 58,6 & 4,8 & 102,4 & 0,15 & 6,36 & 1,91 & 0,86 \\
$\quad$ Estação 2 & & & & & & & & \\
$\quad$ Avicennia schaueriana Staft \& Leechm. & 1,6 & 13,1 & 4,2 & 61,6 & 0,10 & 0,73 & 1,52 & 0,96 \\
$\quad$ L. racemosa & 4,2 & 7,9 & 1,5 & 63,3 & 0,18 & 0,83 & 1,79 & 0,84 \\
$\quad$ R. mangle & 4,9 & 15,7 & 5,0 & 67,2 & 0,05 & 3,54 & 0,81 & 0,43 \\
$\quad$ Estação 3 & & & & & & & & \\
$\quad$ L. racemosa & 2,4 & 13,6 & 1,1 & 72,7 & 0,22 & 0,33 & 1,63 & 0,93 \\
$\quad$ R. mangle & 4,0 & 18,8 & 2,0 & 50,0 & 0,05 & 2,56 & 0,68 & 0,66 \\
$\quad$ Estação 4 & & & & & & & & \\
$\quad$ A. germinans (L.) Stearn. & 1,7 & 37,3 & 4,1 & 77,5 & 0,07 & 1,00 & 3,32 & 1,31 \\
\hline
\end{tabular}

estarem sob maior influência das marés. Os valores descritos para estas estações encontram-se dentro da faixa observada por Vale (1999) que realizou um estudo na desembocadura do rio, onde a salinidade da água intersticial variou entre 28 e 44 . Rhizophora mangle é considerada sal-excludente (Scholander et al. 1962), portanto esperava-se que a água intersticial entre suas raízes exibisse maior teor salino que a das demais espécies, consideradas sal-includentes. Essa tendência foi observada em todas as estações de estudo em que esta espécie esteve presente, apesar de não ter havido diferença significativa entre as espécies.

A matéria orgânica do sedimento é um parâmetro extremamente variável, sujeito ao regime das marés e à produção de serapilheira. Os percentuais médios de matéria orgânica foram considerados baixos, principalmente na estação 1 . Na estação 4 , foi observado o maior percentual deste parâmetro, o que parece refletir o maior desenvolvimento estrutural da floresta de mangue (Silva et al. 2005) e menor influência das marés. De acordo com Lacerda et al. (1995), o material orgânico presente no sedimento de Avicennia schaueriana é mais degradável quando comparado ao de Rhizophora mangle. Dessa forma, a matéria orgânica associada ao sedimento de Avicennia apresentaria taxa mais elevada de decomposição e, conseqüentemente, melhor reciclagem e disponibilidade de nutrientes, conforme observado no presente estudo (estação 2).

Laguncularia racemosa e Rhizophora mangle ocorreram em sedimentos cuja classificação textural variou de arenoso a lama síltico-arenosa, enquanto que Avicennia germinans e Avicennia schaueriana ocorreram em substratos com predominância de sedimentos finos. Estudos têm demonstrado que estas espécies podem se desenvolver em uma variedade de condições, de substratos arenosos a argilosos (Jimenez 1985a,b, Jimenez \& Lugo 1985).

As concentrações de nutrientes do sedimento apresentaram variações inter e intraespecíficas, mas se encontraram dentro da faixa reportada na literatura. Com exceção da estação 1, que exibiu os menores teores de nutrientes, as concentrações de $\mathrm{Ca}, \mathrm{K}$ e $\mathrm{Mg}$ tenderam a diminuir no sentido mar-rio, como um reflexo da fonte marinha destes elementos para o manguezal. Os metais pesados ( $\mathrm{Fe}, \mathrm{Mn}, \mathrm{Zn}$ e $\mathrm{Cu}$ ) não apresentaram esta tendência, visto que são fornecidos para o manguezal através de fontes terrestres, dependendo das características geomorfológicas e climatológicas (Lacerda et al. 1986). Em sedimentos de outros manguezais brasileiros, as concentrações de metais pesados foram muito superiores às obtida no Estuário do Rio São Mateus (Lacerda \& Rezende 1987, Lacerda et al. 1993, Oliveira et al. 1998). Os baixos teores aqui encontrados se devem à ausência de atividades industriais nos arredores do manguezal estudado.

Os menores percentuais de matéria orgânica, as características mais grosseiras do sedimento e os menores teores de nutrientes constatados no sedimento da estação 1 estão relacionados ao acelerado assoreamento verificado nesta estação. Este assoreamento é conseqüência do fato da foz do Rio 
São Mateus apresentar-se instável, com acentuado processo de sedimentação flúvio-marinha, o que vem acarretando problemas de erosão na praia de Conceição da Barra, localizada à margem esquerda, e soterramento da floresta de mangue à margem direita do rio (Vale 1999).

Folhas - Os teores médios determinados para os nutrientes das folhas encontraram-se dentro da faixa descrita na literatura (Lamberti 1969, Lacerda et al. 1986, Cuzzuol \& Campos 2001, Medina et al. 2001, Bernini 2003).

Alguns estudos têm reportado que a descarga de efluentes pode promover um aumento de $\mathrm{N}$ e $\mathrm{P}$ nas folhas (Boto \& Wellington 1983, Clough et al. 1983), pois o sedimento do manguezal é efetivo na retenção de N e P originado de efluentes (Tam \& Wong 1995, 1996, Wong et al. 1997). Próximo à estação 2, ocorre lançamento de efluentes domésticos diretamente no rio ou através da rede pluvial. Entretanto, as conseqüências do lançamento de efluentes sobre o teor de nutrientes nas folhas das árvores de mangue não foram significativas neste estudo.

Avicennia germinans e Avicennia schaueriana destacaram-se pelas maiores concentrações de $\mathrm{N}$ e este comportamento pode ser resultado do acúmulo de glicinbetaína no citoplasma, um composto quaternário de amônio, rico em $\mathrm{N}$, que é considerado um soluto compatível para tolerância aos sais (Popp et al. 1984, Medina \& Francisco 1997). Com relação aos elementos $\mathrm{K}$ e $\mathrm{Mg}$, as maiores concentrações observadas em Avicennia germinans e Avicennia schaueriana, provavelmente refletem a maior permeabilidade a sais nas raízes destas espécies, o que é característico do mecanismo de excreção de sal presente neste gênero (Lacerda et al. 1985, Medina \& Francisco 1997). As duas espécies exibiram baixas concentrações de $\mathrm{Ca}$, as quais refletiram em menores fatores de concentração em relação a Laguncularia racemosa e Rhizophora mangle. Este fato pode ser explicado pela presença de oxalato livre na raiz, que causa a precipitação do $\mathrm{Ca}$ e, possivelmente, evita seu transporte no xilema (Popp 1984, Medina et al. 2001).

Os resultados aqui descritos não confirmaram as indicações de que a absorção de Ca seja controlada pelo nível de $\mathrm{Na}$ no sedimento (Lacerda et al. 1985, 1986), provavelmente, devido aos baixos valores de salinidade. As concentrações das bases trocáveis (K, $\mathrm{Mg}$ e Ca) das folhas não se correlacionaram com as concentrações do sedimento, demonstrando que o acúmulo destes elementos nas folhas não depende da disponibilidade do elemento no sedimento. Resultados semelhantes foram reportados por Lacerda et al. (1985).

No manguezal, os metais pesados podem precipitar na forma de sulfetos, os quais são estáveis sob as condições anóxicas do sedimento e não disponíveis às plantas (Lacerda \& Rezende 1987, Lacerda 1997). Os elementos $\mathrm{Fe}$ e Mn solúveis, precipitados na forma de óxidos, formam placas de ferro na superfície das raízes (Lacerda et al. 1993). Estas características explicam as baixas concentrações de metais pesados no tecido foliar das espécies de mangue. O elemento $\mathrm{Mn}$ não forma sulfetos estáveis, sendo solúvel nas condições anóxicas do sedimento do manguezal, e, portanto mais biodisponível (Aragon et al. 1986, Lacerda et al. 1988).

Houve uma incorporação preferencial de Mn por Rhizophora mangle e de Fe por Laguncularia racemosa, confirmando os resultados descritos para outros manguezais (Lamberti 1969, Lacerda et al. 1985, Cuzzuol \& Campos 2001). Rhizophora é um gênero sal-excludente enquanto que Avicennia e Laguncularia são considerados sal-includentes. Lacerda et al. (1985) demonstraram que o mecanismo de controle de entrada de sal pode afetar a absorção de metais pesados. Dessa forma, o mecanismo de exclusão de sal presente em Rhizophora mangle evitaria a entrada de metais pesados como $\mathrm{Fe}, \mathrm{Zn}$ e $\mathrm{Cu}$, causando menor concentração destes elementos no tecido foliar, como observado neste estudo.

De maneira geral, o acúmulo de $\mathrm{Zn}$ e $\mathrm{Cu}$ nas folhas de Avicennia schaueriana, Avicennia germinans e Laguncularia foi elevado. Resultados similares foram reportados para o manguezal do estuário do Rio Mucuri, Bahia, onde as espécies apresentaram fatores de concentração variando entre 2,2 e 2,6 para Zn e entre 1,7 e 3,2 para Cu (Cuzzuol \& Campos 2001). Lacerda et al. (1986) observaram que ao longo da costa sudeste brasileira, os fatores de concentração para metais pesados em espécies de mangue foram inferiores a 1,0, sendo maiores em Avicennia schaueriana do que em Rhizophora mangle, o que foi em parte confirmado no presente estudo. Os autores demonstraram falta de correlação entre as concentrações de metais nas folhas e no sedimento, fato também observado no manguezal do Estuário do Rio São Mateus. Os baixos valores dos fatores de concentração e a ausência de correlação entre as concentrações das folhas e do sedimento indicam que as plantas de mangue evitam a absorção de metais pesados (Lacerda 1997).

Os resultados descritos neste estudo demonstraram que a concentração de nutrientes do sedimento refletiu a influência da granulometria. Entretanto, o acúmulo dos 
nutrientes nas folhas não refletiu as concentrações do sedimento, confirmando a seletividade de absorção dos íons pelas plantas. Os dados confirmaram o papel do manguezal como barreira biogeoquímica ao trânsito de metais pesados. No entanto, para um melhor entendimento da dinâmica dos nutrientes, torna-se desejável uma amostragem periódica para analisar o efeito sazonal na concentração dos elementos.

Agradecimentos - Ao Instituto Capixaba de Pesquisa, Assistência Técnica e Extensão Rural, Incaper, Linhares, pelas análises das amostras. À Polícia Ambiental de São Mateus e à Prefeitura Municipal de Conceição da Barra, pelo apoio relativo à embarcação. Aos revisores dessa revista, pelas importantes críticas e sugestões. Ao Dr. Carlos Eduardo Rezende e ao MSc. Sérgio Ricardo de Oliveira, pela revisão da primeira versão do manuscrito. As autoras E. Bernini e M.A.B. Silva agradecem ao PIBIC/UFES, pela concessão de bolsa de Iniciação Científica.

\section{Referências bibliográficas}

ALMEIDA, R. 2001. Manguezal do Canal da Passagem, Vitória, Espírito Santo: conteúdo de transferência de nutrientes na fração folhas da serapilheira. Dissertação de mestrado, Universidade de São Paulo, São Paulo.

ARAGON, G.T., PIRES, V.S., LACERDA, L.D. \& PATCHINEELAM, S.R. 1986. Distribuição espacial de nutrientes e metais pesados em sedimentos e águas superficiais em um ecossistema de manguezal. Acta Limnologica Brasiliensia 1:365-385.

BALL, M.C. 1988. Ecophysiology of mangroves. Trees Structure and Function 2:129-142.

BERNINI, E. 2003. Estrutura da vegetação e concentração de nutrientes das folhas e do sedimento das espécies em florestas de mangue do estuário do Rio Paraíba do Sul, Estado do Rio de Janeiro, Brasil. Dissertação de mestrado, Universidade Estadual do Norte Fluminense, Campos dos Goytacazes.

BOTO, K.G. \& WELLINGTON, J.T. 1983. Phosphorus and nitrogen and nutritional status of a northern Australian mangrove forest. Marine Ecological Progress Series 11:63-69.

CINTRÓN, G., LUGO, A.E. \& MARTINEZ, R. 1985. Structural and functional properties of mangrove forests. In The botany and natural history of Panama (W.G. D'Arcy \& M.D.A. Correa, eds.). Missouri Botanical Garden, Saint Louis, p.53-66.

CLOUGH, B.F., BOTO, K.G. \& ATTIWILL, P.M. 1983. Mangrove and sewage: a re-evaluation. In Biology and Ecology of Mangroves (H.J. Teas, ed.). Dr. W. Junk Publishers, Lancaster, v.8, p.151-168.

CUZZUOL, G.R.F. \& CAMPOS, A. 2001. Aspectos nutricionais na vegetação de manguezal do estuário do Rio Mucuri, Bahia, Brasil. Revista Brasileira de Botânica 24:227-234.
EMBRAPACENTRONACIONALDEPESQUISADE SOLOS. 1997. Manual de métodos de análise do solo. Embrapa, Rio de Janeiro.

FITTER, A.H. \& HAY, R.K.M. 1987. Environmental physiology of plants. Academic Press, Oxford.

HAY, J.D. \& LACERDA, L.D. 1984. Ciclagem de nutrientes no ecossistema restinga. In Restinga: origem, estrutura, processos (L.D. Lacerda, D.S.D. Araújo, R. Cerqueira \& B. Turq, eds.). CEUFF, Niterói, p.461-477.

JIMENEZ, J.A. 1985a. Laguncularia racemosa (L.) Gaertn. f. White mangrove. Combretaceae. Combretum family. Unesco, SO-ITF-SM, 3:4p.

JIMENEZ, J.A. 1985b. Rhizophora mangle L. Red mangrove. Rhizophoraceae. Mangrove family. Unesco, SO-ITF-SM, $2: 7 \mathrm{p}$.

JIMENEZ, J.A. \& Lugo, A.E. 1985. Avicennia germinans (L) L. Black mangrove. Avicenniaceae. Verbena family. Unesco-ITF-SM, 4:6p.

LACERDA, L.D. 1986. Pesquisas brasileiras sobre ciclagem de nutrientes em ecossistemas costeiros: identificação de prioridades. Acta Limnologica Brasiliensia 1:3-27.

LACERDA, L.D. 1997. Trace metals in mangrove plantas: why such low concentrations? In Mangrove ecosystem studies in Latin America and Africa (B. Kjerfve, L.D. Lacerda \& H.S. Diop, eds.). Unesco, Paris, p.171-178.

LACERDA, L.D. \& REZENDE, C.E. 1987. Metal geochemistry in mangrove sediments. In Anais do I Simpósio de Ecossistemas Brasileiros (S. Watanabe, coord.). Aciesp, São Paulo, v.3, p.123-131.

LACERDA, L.D., REZENDE, C.E., JOSÉ, D.V., WASSERMAN, J.C. \& FRANCISCO, M.C. 1985. Mineral concentration in leaves of mangrove trees. Biotropica 17:260-262.

LACERDA, L.D., REZENDE, C.E., JOSÉ, D.V. \& FRANCISCO, M.C. 1986. Metallic composition of leaves from the Southeastern Brazilian coast. Revista Brasileira de Biologia 46:395-399.

LACERDA, L.D., MARTINELLI, L.A., REZENDE, C.E., MOZETTO, A.A., OVALLE, A.R.C., VICTORIA, R.L., SILVA, C.A.R. \& NOGUEIRA, F.B. 1988. The fate of trace metals in supended matter in a mangrove creek during a tidal cicle. Science Total Environment 75:249-259.

LACERDA, L.D., CARVALHO, C.E.V., TANIZAKI, K.F., OVALLE, A.R.C. \& REZENDE, C.E. 1993. The biogeochemistry and trace metals distribution of mangrove rizospheres. Biotropica 25:252-257.

LACERDA, L.D., ITTEKKOT, V. \& PATCHINEELAM, S.R. 1995. Biogeochemistry of mangrove soil organic matter: a comparison between Rhizophora and Avicennia soils in South-eastern Brazil. Estuarine, Coastal and Shelf Science 40:713-720.

LAMBERTI, A. 1969. Contribuição ao conhecimento da ecología das plantas do manguezal de Itanhaém. Boletim da Faculdade de Filosofia, Ciências e Letras da Universidade de São Paulo. Botânica 23:1-217. 
LARCHER, W. 2000. Ecofisiologia vegetal. Rima, São Carlos.

LUGO, A.E. \& SNEDAKER, S.C. 1974. The ecology of mangroves. Annual Review of Ecology and Systematic 5:39-64.

MEDINA, E. \& FRANCISCO, A.M. 1997. Osmolaty and d ${ }^{13} \mathrm{C}$ of leaves tissues of mangrove species from environments of contrasting rainfall and salinity. Estuarine, Coastal and Shelf 45:337-344.

MEDINA, E., GIARIZZO, T., MENEZES, M., CARVALHOLIRA, M., CARVALHO, E.A., PERES, A., SILVA, B., VILHENA, R., REISE, A. \& BRAGA, F.C. 2001. Mangal commuties of the "Salgado Paraense": ecologycal heterogneity alone the Bragança peninsula assessed through soil and leaf analisis. Amazoniana 16:397-416.

OLIVEIRA, R.R., BRESSAN, F.A. \& SILVAFILHO, E.V. 1998. Contaminação por metais pesados no sedimento e em compartimentos bióticos de manguezais da Baía de Guanabara, RJ. In Anais do IV Simpósio de Ecossistemas Brasileiros (S. Watanabe, coord.). Aciesp, São Paulo, v.1, p.30-37.

POPP, M. 1984. Chemical composition of Australian mangroves II. Low molecular weigh carbohydrates. Zeitschrift für Pflanzenernahrung und Bodenkunde 113:411-421.

POPP, M., LARHER, F. \& WEIGEL, P. 1984. Chemical composition of Australian mangroves III. Free aminoacids, total methylated onion compounds and total nitrogen. Zeitschrift für Pflanzenernahrung und Bodenkunde 114:15-25.

SALISBURY, F.B. \& ROSS, C.W. 1992. Plant physiology. Wadsworth Publishing Company, Belmont.

SARRUGE, J.R. \& HAAG, H.P. 1974. Análises químicas em plantas. Esalq, Piracicaba.
SCHAEFFER-NOVELLI, Y., CINTRÓN-MOLERO, G., SOARES, M.L.G. \& DE ROSA, T. 2000. Brazilian mangroves. Aquatic Ecosystem Health and Management 3:561-570.

SCHOLANDER, P.F., HAMMEL, H.T., HEMMING-SEN, E. \& GAREY, W. 1962. Salt balance in mangroves. Plant Physiology 37:722-729.

SILVA, D.J. 1981. Análise de alimentos: métodos químicos e biológicos. Imprensa Universitária, Viçosa.

SILVA, C.A.R., LACERDA, L.D. \& REZENDE, C.E. 1990. Heavy metal reservoirs in a red mangrove forest. Biotropica 22:339-345.

SILVA, M.A.B., BERNINI, E. \& CARMO, T.M.S. 2005. Características estruturais de bosques de mangue do estuário do Rio São Mateus, ES, Brasil. Acta Botanica Brasilica 19:465-471.

SUGUIO, K. 1973. Introdução a sedimentologia. Edgar Blucher/Edusp, São Paulo.

TAM, N.F.Y. \& WONG, Y.S. 1995. Mangrove soils as sinks for wastewater-borne pollutants. Hydrobiologia 295:231-241.

TAM, N.F.Y. \& WONG, Y.S. 1996. Retention of wastewaterborne nitrogen and phosphorus in mangrove soils. Environmental Technology 17:851-859.

VALE, C.C. 1999. Contribuição ao estudo dos manguezais como indicadores biológicos das alterações geomórficas do estuário do Rio São Mateus-ES. Dissertação de mestrado, Universidade de São Paulo, São Paulo.

WAISEL, Y. 1972. Biology of halophytes. Academic Press, New York.

WONG, Y.S., TAM, N.F.Y. \& LAN, C.Y. 1997. Mangrove wetlands as wastewater treatment facility: a field trial. Hidrobiologia 352:49-59. 